\title{
A humanização da atenção básica a saúde brasileira no atendimento de travestis e transsexuais: uma revisão narrativa
}

\author{
The humanization of primary care in Brazilian health care for transvestites and \\ transsexuals: a narrative review
}

\section{La humanización de la atención primaria de salud brasileña en la atención de travestis y transexuales: una revisión narrativa}

Lázaro Saluci Ramos ${ }^{1 *}$, Maira Gomes Almeida ${ }^{2}$, Maria Vanderléia Saluci Ramos², Elzinete Maria Carvalho Machado², Valdecy de Oliveira dos Santos ${ }^{2}$, Maria Regina Fontana Contarini ${ }^{2}$, Gleisieres Baiense Fontana Ramos ${ }^{2}$, Tasmânia da Silva Oliveira Mantiolhe ${ }^{2}$, Thiago Favoretti dos Santos Ferreira ${ }^{2}$, Eliangela Nascimento Valim².

\section{RESUMO}

Objetivo: Abordar os desafios da atenção básica a saúde no Brasil e a ausência de humanização em tratamento e acolhimento de travestis e transsexuais. Métodos: Trata-se de análise científica que destaca a escassez de programas que incentivam a humanização e o preparo profissional para o primeiro atendimento nos programas vinculados a atenção primária no Brasil. Tais populações não carece apenas de diagnóstico ou tratamento físico, mas de abordagem humana e comedida. Insurge também a inaptidão da maior parcela dos profissionais que participam do primeiro contato. A linguagem empregada, a diferenciação no tratamento e os múltiplos constrangimentos que podem abalar a confiança desses pacientes e até mesmo afasta-los da égide da saúde pública. Analisar-se-á, também, a necessidade de um ambiente que contemple as novas abordagens clínicas, de modo a eludir o acanhamento e promover maior aceitação por parte do corpo profissional. Conclusão: Apontar as necessidades que cercam a primeira atenção a pacientes transsexuais e travestis e garantir-Ihes tratamento humano e dignificado.

Palavras-chave: Atenção básica, LGBT, Humanização, Estigmas sociais.

\begin{abstract}
Objective: To address the challenges of primary health care in Brazil and the lack of humanization in the treatment and reception of transvestites and transsexuals. Methods: This is a scientific analysis that highlights the scarcity of programs that encourage humanization and professional preparation for the first care in programs linked to primary care in Brazil. Such populations lack not only a diagnosis or physical treatment, but a humane and measured approach. The inability of the majority of professionals who participate in the first contact also rises. The language used, the differentiation in treatment and the multiple constraints that can undermine the confidence of these patients and even keep them from the aegis of public health. The need for an environment that contemplates new clinical approaches will also be analyzed, in order to avoid shyness and promote greater acceptance by the professional body. Conclusion: Point out the needs that surround first attention to transsexual and transvestite patients and guarantee them humane and dignified treatment.
\end{abstract}

Keywords: Primary care, LGBT, Humanization, Social stigmas.

\footnotetext{
${ }^{1}$ Universidade Multivix (MULTIVIX) - Cachoeiro de Itapemirim - ES. *E-mail: lazaro321123@gmail.com

2 Faculdade Vale do Cricaré (FVC) - São Mateus - ES
} 


\section{RESUMEN}

Objetivo: abordar los desafíos de la atención primaria de salud en Brasil y la falta de humanización en el tratamiento y la recepción de travestis y transexuales. Métodos: Este es un análisis científico que destaca la escasez de programas que fomentan la humanización y la preparación profesional para la primera atención en programas vinculados a la atención primaria en Brasil. Dichas poblaciones carecen no solo de un diagnóstico o tratamiento físico, sino de un enfoque humano y medido. La incapacidad de la mayoría de los profesionales que participan en el primer contacto también aumenta. El lenguaje utilizado, la diferenciación en el tratamiento y las múltiples limitaciones que pueden socavar la confianza de estos pacientes e incluso mantenerlos alejados de la salud pública. También se analizará la necesidad de un entorno que contemple nuevos enfoques clínicos, para evitar la timidez y promover una mayor aceptación por parte del cuerpo profesional. Conclusión: Señale las necesidades que rodean la primera atención a los pacientes transexuales y travestis y garantíceles un trato humano y digno.

Palabras clave: Atención primaria, LGBT, Humanización, Estigmas sociales.

\section{INTRODUÇÃO}

A atenção primária a saúde no Brasil desenvolveu inúmeros centros e especialidades para o primeiro atendimento. Evitar que pacientes não tão graves cheguem aos centros de tratamentos mais aprofundados, fez com que a saúde brasileira ficasse menos sobrecarregada. Entretanto, característico do progresso, novos tratamentos, abordagens e atendimentos foram inseridos nessa modalidade (FIOCRUZ, 2009).

No passado recente, profissionais da psicologia integrarem o corpo da saúde pública era considerado distante e desnecessário. As ciências comportamentais eram tratadas com certo desprezo, entretanto nos tempos atuais elas foram incluídas não somente na atenção primária, mas em todos os níveis da saúde pública, inclusive nos ambientes educacionais, laborais e carcerários (BOING E, CREPALDI MA, 2010).

Boff $L$ (2002) fez uma abordagem de cuidado que muito se espera no sistema público de saúde, para ele cuidado é uma atividade de zelo, desvelo e responsabilidade para com o outro. Da mesma forma, faz-se necessário preparar os profissionais da atenção básica a saúde brasileira para o cuidado e o acolhimento. Introduzir na formação dos profissionais a humanização, e garantir aos profissionais sem formação acadêmica uma extensão em acolhimento.

Para melhor compreensão, cabe diferenciar as terminologias supracitadas. Travesti é um termo muito utilizado em países da América Latina, Portugal e Espanha para designar pessoas com identidade de gênero feminina que não se incomodam com a genitália tipicamente do sexo masculino. Já a transexualidade não se refere apenas a identidade de gênero feminina, mas qualquer pessoa que não se sinta adequada ao gênero que se encontra e por isso realize a adequação do próprio corpo ao novo gênero. Ambas as definições carregam significados amplamente discutíveis e por isso não podem ser tratadas como regra apenas pelos seus sentidos gramaticais. A língua portuguesa é um idioma vivo, que inova os sentidos das palavras de acordo com suas adequações (COELHO MTAD, SAMPAIO LLP, 2014).

Desde o acolhimento até o diagnóstico, o atendimento a essas populações costuma ser extremamente constrangedor. O vocabulário costumeiramente empregado, fruto da transfobia e da travestifobia histórica, as diferentes necessidades nas abordagens clínicas e a desconstrução de preconceitos como a sorofobia, que é a discriminação contra pessoas soropositivas ou com populações mais vulneráveis a doença.

É Necessário introduzir na atenção primária brasileira políticas públicas com o objetivo de preparar os profissionais e conscientizar a comunidade, já que o ambiente onde se promove a saúde pública é constantemente frequentado por grandes grupos de pessoas, que através de gestos e atos, podem inibir a aproximação dos grupos objetivos de tais políticas públicas (COELHO MTAD, SAMPAIO LLP, 2014).

O objetivo do presente estudo é abordar as deficiências do Sistema público de saúde brasileiro no atendimento de travesties e transsexuais, e buscar soluções eficazes e rápidas para solucionar a ausência 
de políticas públicas em tal área, afim de aproximar a população excluída dos programas de saúde da família, e garantir o primeiro acolhimento quando necessário

\section{REVISÃO BIBLIOGRÁFICA}

Para o Ministério da Saúde a Atenção Básica tem a Saúde da Família como porta de entrada para o desenvolvimento e aplicação dos objetivos do SUS: garantir o acesso universal e contínuo a básicos de saúde pública, resguardados como a passagem preferível do sistema de saúde, com área adscrito de forma a possibilitar a organização descentralizada, e em sintonia com o princípio da equidade; garantir e integrar em suas várias vias, a propor: integração de programas a necessidades espontâneas; programação de ações da área da saúde, redução de novos danos, vigilância à saúde, tratamento e reabilitação, inclusão e desenvolvimento de atividades conjuntas, e coordenação do cuidado na rede de serviços; construir relações de confiança e planejamento coletivo dentro das equipes e a população citada, efetivando a continuidade das ações de saúde e a longitudinalidade do cuidado; valorizar o corpo de profissionais através de melhores condições de trabalho e do ampliação constante nas ações de formação e capacitação; avaliar e observar todos os resultados apontados como meio de melhoramento, garantir sempre a participação popular e avaliação da comunidade (BRASIL, 2017).

A atenção básica envolve inúmeros outros programas que cercam as comunidades. A estratégia e saúde da família por exemplo, é um dos programas mais antigos da atenção básica, onde as populações vulneráveis chegam no princípio de suas enfermidades ou buscam diagnósticos rotineiros no intuito de prevenir doenças. As unidades de saúde e os pontos de apoio distribuídos pelo Brasil são as principais casas dos programas de saúde pública (FIOCRUZ, 2009).

Os campos de atuação da saúde pública dentro da Atenção Básica são bem vastos, ela abrange a promoção e a proteção da saúde, a prevenção de agravos, o diagnóstico, o tratamento, a reabilitação e a manutenção da saúde. É desenvolvida por meio do exercício de práticas gerenciais e sanitárias democráticas e participativas, sob forma de trabalho em equipe, dirigidas a populações de territórios bem delimitados, pelas quais assume a responsabilidade sanitária, considerando a dinamicidade existente no território em que vivem essas populações (TANAKA OY, 2011). Segundo o Decreto 8.727, de 28 de Abril de 2016:

"Dispõe sobre o uso do nome social e o reconhecimento da identidade de gênero de pessoas travestis e transexuais no âmbito da administração pública federal direta, autárquica e fundacional [...] Art. 2ㅇ Os órgãos e as entidades da administração pública federal direta, autárquica e fundacional, em seus atos e procedimentos, deverão adotar o nome social da pessoa travesti ou transexual, de acordo com seu requerimento e com o disposto neste Decreto [...] Art. 6o $A$ pessoa travesti ou transexual poderá requerer, a qualquer tempo, a inclusão de seu nome social em documentos oficiais e nos registros dos sistemas de informação, de cadastros, de programas, de serviços, de fichas, de formulários, de prontuários e congêneres dos órgãos e das entidades da administração pública federal direta, autárquica e fundacional (BRASIL, 2016). Parágrafo único. É vedado o uso de expressões pejorativas e discriminatórias para referir-se a pessoas travestis ou transexuais."

Geralmente os programas da atenção básica são compostos, no que tange ao corpo profissional, de médicos, enfermeiros, dentistas, agentes comunitários de saúde, auxiliares administrativos, atendentes, psicólogos, assistentes sociais e outros especialistas na finalidade de atender todas as suas demandas. Todos não possuem nenhum preparo para atender um caso específico ou abordar de maneira sistêmica e profissional, uma pessoa transsexual ou travesti (DIAS FX, SILVA LCA, 2016).

Mesmo a Carta dos Direitos dos Usuários do SUS ter sido promulgada em 2006 garantindo que todos os cidadãos têm direito a humanização no atendimento, com acolhimento e livre de qualquer margem preconceituosa (Brasil, 2006), estes paradigmas estão muito longe de serem quebrados.

Em uma pesquisa feita com 15 pessoas trans, foram relatados aos entrevistadores casos que indicavam fortes sentimentos de angústia e tristeza diante da discriminação vivenciada nos serviços disponibilizados 
pela saúde pública brasileira, o que indica descaso com os tratamentos em andamento e repulsa na procura por novos cuidados a saúde quando precisos (ROCON PC, et al., 2019).

No que concerne à saúde sicológica, o descaso à identidade de gênero é identificado nos discursos como um dos maiores causadores de distúrbios psíquicos, como é claro na fala de pessoas abordadas em averiguações, quando elas relatam o não reconhecimento da sua identidade de gênero the causa dor e sofrimento, tanto físico como psicológico. Pesquisas indicam que a maior vulnerabilidade a transtornos mentais neste público é relacionada ao o meio social em que vivem, cercado de estigmas, discriminações, preconceitos e exclusões, o que coloca em risco a saúde mental e amplia a incidência de tentativas de suicídio (CORTES HM, et al., 2019).

Garantir a humanização, nos demais sentidos apontados mais objetivamente no caso da saúde, requer aceitar e reconhecer que nessa área e nas suas didáticas, em especial, existem grandes problemas e carências de muitas das condições exigidas pela definição da concepção, organização e implementação do cuidado da saúde da humanidade, tanto por parte dos organismos e práticas do Estado, como da sociedade (OLIVEIRA BRG, et al., 2006).

\section{Acolhimento e abordagem}

Desde os primórdios, a comunicação se tornou nossa maior ferramenta de progresso. O homem evoluiu em quase todos os sentidos através da escrita. Até então, grandes desafios foram lançados no campo da comunicação e dentre eles, como se referir as diferenças sem ser ofensivo. Ou em alguns casos, até mesmo como ofender sem parecer arrogante (CENDÓN BV, 2001).

Martino LC (2002), trata o ser humano como um ser de comunicação com base na sua criação e na inserção de noções desde tenra idade. Para o autor, o ser humano não tem escolha senão ser comunicativo, é a única alternativa que lhe é dada, embutindo no seu conceito a ideia de relação: "No caso humano, nós não temos senão relações".

A comunicação nos permite, dentre tantas benesses, aproximar ou afastar seres dotados de racionalidade ou instinto. $O$ acolhimento parte dessa premissa, através da comunicação, seja ela oral ou física. E a abordagem correta para o acolhimento requer conhecimento rigoroso da língua e seus inúmeros pronomes de tratamento. O manuseio de um simples instrumento pode indicar rejeição, aceitação, acolhimento ou intenção adversa (JACQUES MGC, et al., 2008).

A substituição de termos como o homossexualismo onde o sufixo "ismo" indica patologia, por homossexualidade ou homoafetividade, representa um grande avanço em questões de representatividade e respeito para a população de Lésbicas, Gays, Bissexuais, Transsexuais, Travestis e Intersexuais (LGBTI) (GORISGH PCVS, 2017).

De tal forma, o acolhimento e a abordagem não é nada menos que resguardar o direito inviolável a saúde. Assegurar o acesso sem restrições a transsexuais e travestis nos centros públicos da atenção básica é respeitar a garantia do principal bem jurídico que dispõe a Constituição Federal de 1988, a vida. Independente se viola valores morais familiares, tradicionais ou conceitos religiosos, nenhum desses entendimentos pode se sobrepor as individualidades humanas e a pluralidade de condições (ROCON PC, et al., 2019). Segundo o Art. 196:

"A saúde é direito de todos e dever do Estado, garantido mediante políticas sociais e econômicas que visem à redução do risco de doença e de outros agravos e ao acesso universal e igualitário às ações e serviços para sua promoção, proteção e recuperação (BRASIL, 1988) ".

Humanizar, instruir e acolher, essas são as melhores maneiras de introduzir travestis e transsexuais nos programas da atenção básica. O saber acolher é a mais eficaz ferramenta para concretizar esse objetivo. Um olhar que indique estranhamento e até mesmo uma palavra mal-empregada, pode ser o suficiente para distanciar o paciente ou tornar a equipe incapaz de aferir as suas necessidades. Em 2008 o núcleo técnico de política nacional e humanização, publicou um periódico direcionado ao público masculino, que é um grupo que ainda é muito relapso quando o assunto é cuidado com a saúde. A semelhança se dá quando este grupo 
também é vitimado por um preconceito, o machismo estrutural. No periódico foi apresentada uma nova política pública do governo federal que buscava atrair o público masculino aos cuidados da atenção básica (ROSA DF, et al., 2019).

Essa política envolve cuidados tanto da Atenção Básica com foco na Estratégia de Saúde da Família, quanto nos demais níveis de atenção, no que diz respeito à promoção, prevenção, assistência e recuperação da saúde, além de chamar a atenção para a responsabilidade dos três níveis de gestão, na prática da humanização, integração das demais políticas, articulação intersetorial, reorganização das ações de saúde, estímulo a corresponsabilidade de entidades da sociedade organizada, aperfeiçoamento do sistema de informações em saúde e realização de estudos e pesquisas a respeito da saúde do homem (BRASIL, 2008).

A exemplo dessas políticas públicas direcionadas a saúde do homem, políticas públicas, tanto no campo do aperfeiçoamento para os integrantes do corpo profissional dos centros da atenção básica, quanto para informar o público alvo da disponibilidade e oferta, podem ser desenvolvidas para a população de travestis e transsexuais. Incluindo também a conscientização da comunidade quanto a importância de ter este público nos aconchegos da saúde pública (SILVA LKM, et al., 2017).

Além da ausência de comunicação adequada, há de se falar também da sorofobia. A sorofobia é o preconceito contra pessoas soropositivas ou grupos que estão mais vulneráveis a doença. A população de Lésbicas, Gays, Bissexuais, Transsexuais, Travestis e Intersexuais (LGBTI) foi colocada a margem da sociedade quando o assunto é HIV e AIDS.

Segundo pesquisadores do Imperial College e da London School of Hygiene and Tropical Medicine, o risco de transmissão de HIV pelo sexo anal é dezoito vezes maior que no sexo vaginal. Isso coloca parcela do grupo dos transsexuais, travestis e gays em um grupo de maior risco quando o assunto é a contaminação (DOURADOS, 2018).

Com base em dados e informativos nesse sentido, criou-se a falsa ideia de que a população LGBTI deve ser colocada sob suspeita ou até mesmo distanciada da comunidade por se tratar de grupo de alto risco. Muito comum entre os brasileiros o equívoco quanto as formas de transmissão da doença, por isso, como forma de se prevenir, a comunidade acaba isolando os pacientes infectados e os grupos vulneráveis (DOURADOS, 2018).

O HIV é um vírus que agride o sistema de imunidades do ser humano, ele faz com que o organismo se torne vulnerável a doenças parasitárias. Isto devido a agressividade com que ele acaba com as células de defensoras, levando abaixo a imunidade corporal e levando ao aparecimento da AIDS (FERNANDES N, 2017).

Este preconceito também é comum no corpo profissional dos ambientes de saúde, em quase todas as vezes nota-se o despreparo do profissional e a ausência de informação. Olhares, excesso de cuidados e outras formas de demonstrar o medo ou a rejeição pelo paciente pode acabar se tornando mais um motivo de distanciamento do público alvo da atenção básica (ROSA DF, et al., 2019).

Compreender que o preconceito afasta o paciente e aumenta o problema social é o primeiro caminho para buscar expungir da saúde pública a sorofobia. Preparar o corpo profissional e ampliar as campanhas de conscientização contra o preconceito é uma necessidade urgente. Apesar do Brasil ser o País com melhores resultados nas campanhas de combate ao preconceito com pessoas soropositivas, o preconceito social ainda é grande (UNAIDS, 2019).

\section{Tratamentos específicos}

Assim como as mulheres necessitam de cuidados diferentes do homem quando se trata de saúde do corpo, transsexuais e travestis também requerem cuidados específicos em alguns casos. Uma mulher transsexual ou uma travesti necessitam ainda de realizar por exemplo o exame de toque retal para averiguar alguma alteração na próstata. Mesmo se tratando de algo raríssimo, o câncer de próstata em pessoas trans e travestis que realizam tratamento hormonal. Assim como o homem transsexual também deve atentar-se aos cuidados com o útero e com os seios, mesmo sendo reduzido o número de casos de câncer de mama em pessoas mastectomizadas (DRAUZIO, 2015). 
Inúmeros são os casos distintos que podem levar essas populações a procurar os centros da atenção básica. E humanizar a estrutura desses centros, além de garantir o preparo das equipes profissionais, faz-se mister. Quantos profissionais da saúde pública estariam preparados hoje para não constranger uma transsexual ou uma travesti que deseja informações acerca dos cuidados com seu órgão genital? Quantos profissionais estariam preparados para realizar sem constranger em um homem transsexual um exame preventivo? Ou um homem transsexual que deseja informações sobre o exame de mama para diagnosticar um possível câncer? (GUERRERO P, et al., 2013).

Outro tratamento específico das populações transsexuais, travestis e também das mulheres, são os tratamentos hormonais. Apesar de não ser um tratamento oferecido através da atenção básica no Brasil, as primeiras informações ou fases do tratamento podem ser realizados através dela. Cabe ressaltar também que além do constrangimento, muitos profissionais não estariam preparados adequadamente para tal demanda. (GUERRERO P, et al., 2013).

Nas duas décadas que se passaram, houveram diálogos entre o poder público federal e a sociedade civil organizada, mudanças interessantes foram propostas e muitas delas alcançadas para aprimorar o sistema público brasileiro de saúde. Dentre algumas, destacam-se a formulação do Plano Nacional de Combate à Violência e à Discriminação de Lésbicas, Gays, Bissexuais e Travestis, de 2004, que aprecia atos de ampliação e promoção de direitos, inclusão internacional, educação, segurança, trabalho e saúde; a Proposta dos Direitos de Usuários da Saúde, de 2006, que inclui o direito a identidade de gênero com nome social em qualquer casa da atenção básica ou do SUS; o Plano de Enfrentamento da Aids entre Gays, HSH e Travestis, de 2007; as regulamentações de 2008 e 2013 acerca do processo transexualizador no âmbito do SUS, que inserem a cirurgia de redesignação sexual, a ampliação do cuidado com transexuais; e a Política Nacional de Saúde Integral de Lésbicas, Gays, Bissexuais e Travestis e Transexuais, de 2010 (PELÚCIO L, 2009).

Desde 2004 a saúde da população LGBTI vem sendo discutida nas conferências nacionais de saúde. Muitas pautas foram abordadas, elaboradas, apresentadas, aperfeiçoadas e inseridas na saúde pública (SAMPAIO AF, 2015). Entretanto, nota-se o desprezo constante com as pautas ligadas a transexualidade e a travestilidade. Alguns grupos do alfabeto LGBTI seguem invisíveis ou impossibilitados de ampla representação (CHAGAS EM, NASCIMENTO TEP, 2017).

É indiscutível que há um enorme despreparo da saúde pública brasileira no atendimento de transsexuais e travestis. Os estigmas sociais acabam desencadeando uma espécie de bola de neve que bloqueia a procura desses públicos por programas preventivos de saúde. Humanizar a saúde pública garantirá um diagnóstico mais eficaz, um atendimento mais conciso e uma inserção mais rápida de populações em situação de vulnerabilidade nos centros da atenção básica (NORA CRD, JUNGES JR, 2013).

\section{CONSIDERAÇÕES FINAIS}

Conclusas as análises dos processos de humanização e aperfeiçoamento no atendimento da atenção básica brasileira a travestis e transsexuais, chega-se ao entendimento que a forma mais eficaz de preparar este ambiente para receber novos públicos é incluir uma política nacional de humanização através das pautas dos direitos humanos. Requer também, que a comunidade seja inteirada da importância de não permitir que um grupo vulnerável cresça nessa situação. As legislações recentes de combate a LGBTIfobia devem ser utilizadas para coibir casos de preconceito ou abordagens inadequadas de membros da comunidade. De acordo com decisão do Supremo Tribunal Federal do ano de 2019, os crimes de ódio contra qualquer membro da comunidade LGBTI serão punidos com a legislação que pune os crimes de racismo.

\section{REFERÊNCIAS}

1. BOING E, CREPALDI MA. O Psicólogo na atenção básica: uma incursão pelas políticas públicas de saúde Brasileiras. Psicologia: Ciência e Profissão, 2010; 30(9): 133-139.

2. BOFF L. Saber cuidar: ética do humano, compaixão pela terra. Petrópolis: Vozes, 2002.

3. BRASIL. 1988. Constituição da República Federativa do Brasil: promulgada em 5 de outubro de 1988. 4. ed. São Paulo: Saraiva, 1990. 
4. BRASIL. 2008. Acolhimento nas práticas de produção de saúde/Ministério da Saúde, Secretaria de Atenção à Saúde, Núcleo Técnico da Política Nacional de Humanização. - 2. ed. - Brasília: Editora do Ministério da Saúde.

5. BRASIL. 2017. In: MINISTÉRIO DA SAÚDE. Disponível em: http://www.saude.gov.br/artigos/770-sistema-nacionalde-saude/40315-atencao-basica . Acesso em: 11 de out. 2019.

6. CENDÓN BV. Ferramentas de busca na Web. Ciência da Informação, 2001; 13(3): 59-63.

7. CHAGAS EM, NASCIMENTO TEP. (IN)VISIBILIDADE TRANS: uma breve discussão acerca da transfobia na vida de travestis e transexuais. VIII jornada internacional políticas públicas, 2017.

8. COELHO MTAD, SAMPAIO LLP. Transexualidades, um olhar multidisciplinar. 2nd ed. Salvador: edufba, 2014; $98 \mathrm{p}$.

9. CORTES HM, et al. Vivências de mulheres transgêneras de um município do recôncavo da

10. Bahia, Revista Eletrônica Acervo Enfermagem. Vegetatio, 2019: 1-9.

11. DIAS FX, SILVA LCA. Percepções dos Profissionais sobre a Atuação dos Psicólogos nas Unidades Básicas de Saúde. Psicologia: Ciência e profissão, 2016; 4(7): 333-339.

12. DOURADOS, 2018. In: DOURADOS NOTÍCIAS. Disponível em: https://www.douradosagora.com.br/noticias/cienciae-saude/risco-de-transmissao-do-hiv-no-sexo-anal-e-18-vezes-maior-que-no-vaginal. Acesso em: 12 de nov. 2019.

13. DRAUZIO. 2015. In: Transexuais femininas precisam fazer exame de próstata? Brasil: Uol. Disponível em: $\mathrm{https://drauziovarella.uol.com.br/entrevistas-2/transexuais-femininas-precisam-fazer-exame-de-prostata/.} \mathrm{Acesso}$ em: 14 dez. 2019.

14. FERNANDES N. Síndrome do preconceito: como o estigma contribui para o aumento da epidemia de aids. São Paulo: Galileu, 2017; 27p.

15. GUERRERO $P$, et al. $O$ acolhimento como boa prática na atenção básica à saúde. Texto \& Contexto - Enfermagem, 2013; $22: 11-19$.

16. FIOCRUZ. 2009. In: DICIONÁRIO DA EDUCAÇÃO PROFISSIONAL EM SAÚDE. Disponível em; http://www.sites.epsjv.fiocruz.br/dicionario/verbetes/ateprisau.html . Acesso em; 14 de dezembro de 2019.

17. GORISGH PCVS. O reconhecimento dos direitos humanos lgbt: de stonewall à onu (Direitos Humanos e Inclusão). Appris, 2017.

18. JACQUES MGC, et al. Relações Sociais e Ética. 3nd ed. Rio de Janeiro: SciELO Books, 2008; $198 p$.

19. MARTINO LC. De qual comunicação estamos falando? Teorias da comunicação: conceitos, escolas e tendências. Petrópolis: Vozes, 2001, 31p.

20. NORA CRD, JUNGES JR. Política de humanização na atenção básica: revisão sistemática. Revista de saúde pública, 2013.

21. OLIVEIRA BRG, et al. A humanização na assistência à saúde. Rev. Latino-Am. Enfermagem vol.14 no.2, 2006 .

22. PELÚCIO L. Abjeção e desejo uma etnografia travesti sobre o modelo preventivo de AIDS. São Paulo: Annablume; 2009.

23. ROCON PC, et al. Acesso à saúde pela população trans no Brasil: Nas entrelinhas da revisão integrativa. Trabalho, educação e saúde, 2019; 18: 101-105.

24. ROSA DF, et al. Assistência de Enfermagem à população trans: gêneros na perspectiva da prática profissional, Revista Brasileira de Enfermagem. Brasília, 2017; 33: 87-96

25. SAMPAIO AF. Saúde da população LGBT: O Círculo de Cultura na construção de saberes de profissionais da Atenção Básica. Revista eletrônica UERN, 2015.

26. SILVA LKM, et al. Uso do nome social no Sistema Único de Saúde: elementos para o debate sobre a assistência prestada a travestis e transexuais. Revista de Saúde Coletiva, 2017; 26: 42-48.

27. TANAKA OY. Avaliação da atenção básica em saúde: uma nova proposta. Saúde e sociedade, 2011; 2(12): $33-41$.

28. UNAIDS. 2019. In: UNAIDS.ORG. Estudo revela como o estigma e a discriminação impactam pessoas vivendo com HIV e AIDS no Brasil. Disponível em: https://unaids.org.br/2019/12/estudo-revela-como-o-estigma-e-a-discriminacaoimpactam-pessoas-vivendo-com-hiv-e-aids-no-brasil/ . Acesso em: $11 \mathrm{de}$ dez. 2019. 